Supporting Information to

Unraveling Mass and Electron Transfer Kinetics at Silica

Nanochannel Membrane Modified Electrodes by Scanning

\title{
Electrochemical Microscopy
}

Lina Yao, Kexin Chen, Bin Su*

Institute of Analytical Chemistry, Department of Chemistry, Zhejiang University,

Hangzhou 310012, China

* Corresponding Authors:

Prof. Bin Su, Email: subin@zju.edu.cn

Table of Content

S1. COMSOL Simulations

S2. Characterization of SNM/ITO Electrode

S3. Calculation of SNM permeability using effective medium theory

Appendix. Comsol Model Report 


\section{S1. COMSOL Simulations}

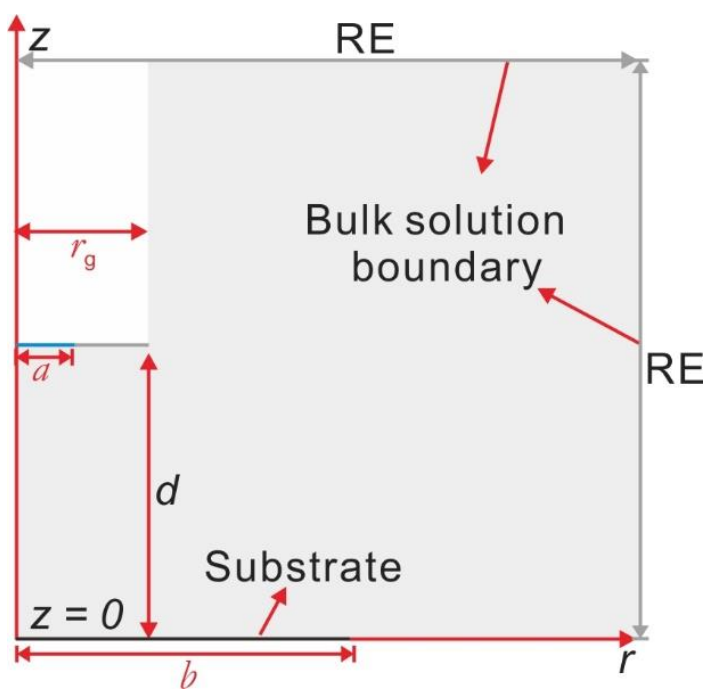

Figure S1. Two-dimensional (2D) axisymmetric model with labeled boundaries for simulating mass transfer process across nanoporous silica membrane (not to scale).

The diffusion problem of redox probe through SNM was simulated using COMSOL Multiphysics (version 5.2). A two-dimensional axisymmetric model is applied for the simulation and the schematic geometry model is shown in Figure S1 with labeled boundaries. RE represents the simulation limit (bulk solution boundaries) and is defined to be $200 \times$ of the UME radius $(a=3.5 \mu \mathrm{m})$. The size of substrate is defined as $100 \times$ of $a$ to represent the infinite substrate relative to UME radius.

Considering a simple one-electron oxidation at the tip,

$$
\mathrm{R}-e^{-} \rightarrow \mathrm{O}
$$

The steady-state diffusion of $\mathrm{R}$ and $\mathrm{O}$ in solution can be expressed as,

$$
\frac{\partial c_{i}(r, z)}{\partial t}=D_{i}\left[\frac{\partial^{2} c_{i}(r, z)}{\partial r^{2}}+\frac{1}{r} \frac{\partial c_{i}(r, z)}{\partial r}+\frac{\partial^{2} c_{i}(r, z)}{\partial z^{2}}\right]=0
$$

where $c_{i}(r, z)$ and $D_{i}$ are the concentration and diffusion coefficient of species $i(i=\mathrm{O}, \mathrm{R})$, respectively. $r$ and $z$ refer to the directions parallel and normal to the UME and substrate surface, respectively. The diffusion coefficients of $\mathrm{O}$ and $\mathrm{R}$ are assumed to be equal for simplicity. Under this condition, the local mass conservation is always maintained in the 
simulation domain,

$$
c_{\mathrm{O}}(r, z)=c_{\mathrm{R}}^{0}-c_{\mathrm{R}}(r, z)
$$

where $c_{\mathrm{R}}^{0}$ is the initial concentration of $\mathrm{R}$. The diffusion problem can then be described in terms of only one species, R. Thus, the boundary conditions are expressed as,

$$
\begin{aligned}
& c_{\mathrm{R}}(r, d)=0, \quad 0<r<a, \quad t>0 \\
& {\left[\frac{\partial c_{\mathrm{R}}(r, z)}{\partial z}\right]_{z=d}=0, \quad a<r<r_{\mathrm{g}}} \\
& {\left[\frac{\partial c_{\mathrm{R}}(r, z)}{\partial r}\right]_{r=0}=0, \quad 0<z<d} \\
& c_{\mathrm{R}}(r, z)=c_{\mathrm{R}}^{0}, \quad r_{\mathrm{g}}<r<R E, z=R E ; r=R E, 0<z<R E
\end{aligned}
$$

where $r_{\mathrm{g}}$ is the radius of outer insulating sheath surrounding the tip and RE the dimension of simulation space limit (see details in Figure S1). The initial condition is,

$$
c_{\mathrm{R}}(r, z)=c_{\mathrm{R}}^{0}, \quad t=0
$$

Considering an irreversible substrate reaction, the surface flux boundary condition for bare ITO electrode is given by

$$
J==k_{\mathrm{f}}\left(c_{\mathrm{R}}^{0}-c_{\mathrm{R}}(r, 0)\right)
$$

For SNM modified ITO electrode, namely SNM/ITO, the surface flux boundary condition is derived in the THEORY section in the main manuscript, and is given by

$$
J=k_{\text {app }}\left(c_{\mathrm{R}}^{0}-c_{\mathrm{R}}(r, 0)\right)
$$

where $k_{\text {app }}$ is defined by eq. 15 .

In the SG/TC mode, $\mathrm{R}$ is oxidized to $\mathrm{O}$ at the ITO surface and regenerated at UME tip under diffusion-limited condition. The reactions can be expressed as, 


$$
\begin{aligned}
& \mathrm{O}+e^{-} \rightarrow \mathrm{R} \quad \text { (at the tip) } \\
& \mathrm{R}-e^{-} \rightarrow \mathrm{O} \quad \text { (at the substrate) }
\end{aligned}
$$

Despite of the transient current obtained at the large substrate applied in this mode $(d=5$ $\mathrm{mm}$ ), we assume that the tip reaction is at the steady-state, considering the diffusion cycling of $\mathrm{O} / \mathrm{R}$ couple between the substrate and tip at the long time scale. The boundary conditions can be summarized as,

$$
\begin{aligned}
& c_{\mathrm{O}}(r, d)=0, \quad 0<r<a \\
& {\left[\frac{\partial c_{\mathrm{R}}(r, z)}{\partial z}\right]_{z=d}=\left[\frac{\partial c_{\mathrm{O}}(r, z)}{\partial z}\right]_{z=d}=0, \quad a<r<r_{\mathrm{g}}} \\
& {\left[\frac{\partial c_{\mathrm{R}}(r, z)}{\partial r}\right]_{r=0}=\left[\frac{\partial c_{\mathrm{O}}(r, z)}{\partial z}\right]_{z=d}=0, \quad 0<z<d} \\
& c_{\mathrm{R}}(r, z)=c_{\mathrm{R}}^{0}, c_{\mathrm{O}}(r, z)=0, r_{\mathrm{g}}<r<R E, z=R E ; r=R E, 0<z<R E \\
& {\left[\frac{\partial c_{\mathrm{R}}(r, z)}{\partial z}\right]_{z=0}=-\left[\frac{\partial c_{\mathrm{O}}(r, z)}{\partial z}\right]_{z=0}}
\end{aligned}
$$

The tip current is obtained by integrating the flux of redox probe to the carbon fiber surface. The current can be described by the following equation,

$$
i=2 \pi n F D \int_{0}^{a} r\left[\frac{\partial c_{\mathrm{R}}(r, z)}{\partial z}\right] \mathrm{d} r
$$

where $n$ is the number of electrons transferred in the tip reaction ( $n=1$ in this work), $F$ is the Faraday constant, $D$ is the diffusion coefficient of redox probe and $a$ is the tip radius $(3.5 \mu \mathrm{m})$.

The COMSOL model report in attached further below as the appendix. 


\section{S2. Characterization SNM/ITO Electrode}

SNM modified ITO electrode was firstly characterized by cyclic voltammetry. $\mathrm{Ru}\left(\mathrm{NH}_{3}\right)_{6}{ }^{3+}$, $\mathrm{FcMeOH}$ and $\mathrm{Ru}(\mathrm{CN})_{6}{ }^{4}$ were used as probe molecules. When $\mathrm{CTAB}$ micelles were retained in nanochannels (Figure S2a), no faradic currents were observed for charged $\mathrm{Ru}\left(\mathrm{NH}_{3}\right)_{6}{ }^{3+}$ and $\mathrm{Ru}(\mathrm{CN})_{6}{ }^{4-}$ in both $1.0 \mathrm{M}$ and $0.01 \mathrm{M} \mathrm{KCl}$ solution while a pair of obvious redox peaks are observed for $\mathrm{FcMeOH}$ and the peak current varies moderately when decreasing $\mathrm{KCl}$ concentration from 1.0 M to 0.01 M. After CTAB removal (Figure S2b), all three probes show obvious faradic behavior in $1.0 \mathrm{M} \mathrm{KCl}$. At lower concentration of $\mathrm{KCl}$, redox peaks of negatively charged $\mathrm{Ru}(\mathrm{CN})_{6}{ }^{4}$ disappear (Figure S2b, black solid line), indicating that no $\mathrm{Ru}(\mathrm{CN})_{6}{ }^{4}$ is reacted at $\mathrm{SNM} / \mathrm{ITO}$ interface. These results qualitatively demonstrate that CTAB@SNM repulse charged species and that SNM selectively permit the access of anions dependent on the electrolyte concentration.

a)

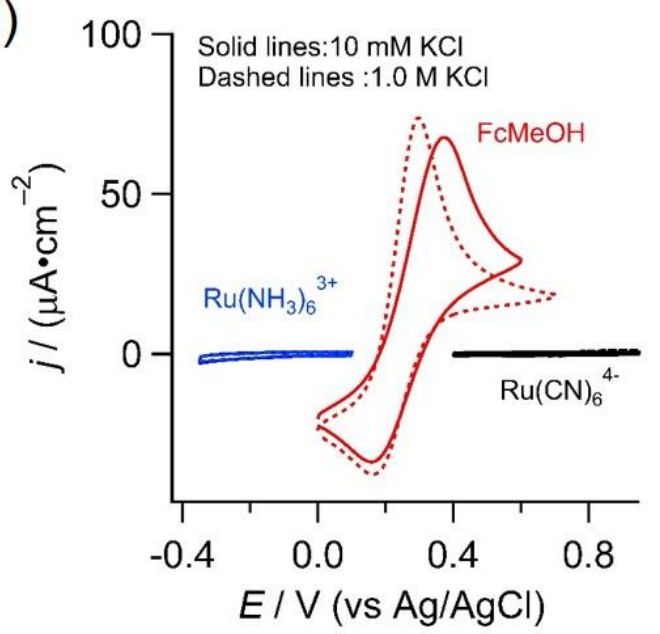

b)

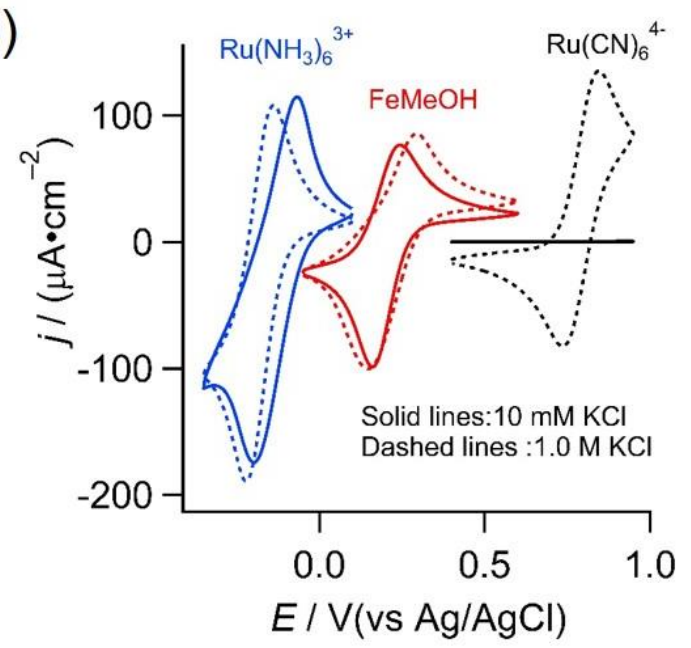

Figure S2. Cyclic voltammetry of CTAB@SNM/ITO (a) and SNM/ITO (b) electrodes in $1.0 \mathrm{M}$ and $0.01 \mathrm{M} \mathrm{KCl}$ solution containing different redox probes: $1 \mathrm{mM} \mathrm{Ru}\left(\mathrm{NH}_{3}\right)_{6}{ }^{3+}$ (blue curves), 1 $\mathrm{mM} \mathrm{FcMeOH}$ (red curves) and $1 \mathrm{mM} \mathrm{Ru}(\mathrm{CN})_{6}{ }^{4}$ (black curves).

SEM and TEM images of SNM were captured on a SU8010 scanning electron microscope and an HT7700 transmission electron microscope, respectively. Figure S3a shows the cross-sectional SEM image of SNM, from which a three-layer structure, namely SNM, ITO and glass layer from top to bottom, can be clearly resolved. The thickness of the 
SNM membrane is measured to be $97.5 \mathrm{~nm}$. Figure S3b is the top view TEM image of SNM, displaying the distribution of the pores (bright dots, $2.3 \mathrm{~nm}$ in diameter. The right inset of Figure S3b is a cross-sectional TEM image of SNM nanochannels with a length of $97.2 \mathrm{~nm}$, which is in accordance with that measured in SEM (Figure S3a).
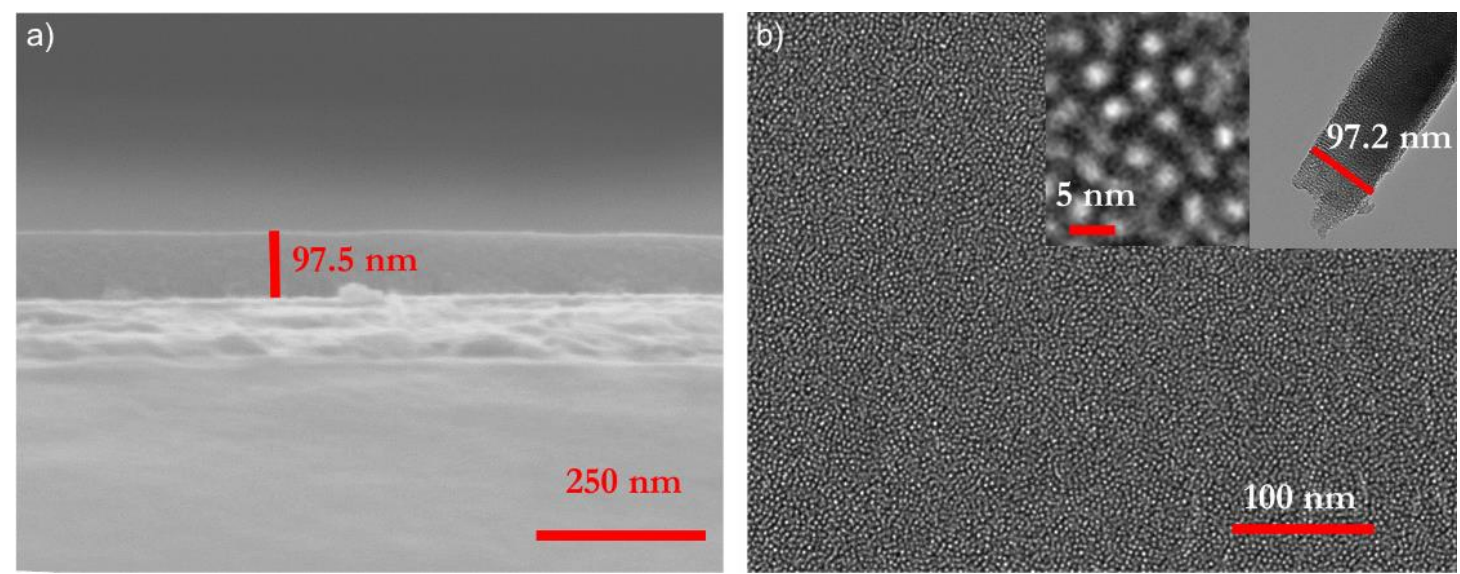

Figure S3. (a) The cross-sectional SEM image of SNM/ITO glass. (b) The top view TEM image of SNM. The insets: the magnified top view TEM image with a scale bar of $5 \mathrm{~nm}$ (left) and crosssectional TEM image of SNM. 


\section{S3. Calculation of SNM Permeability Using Effective Medium Theory}

According to the effective medium theory, the permeability and structural properties of a nanoporous membrane can be related by,

$$
k_{\mathrm{m}}=2 N D_{\mathrm{w}} r /(2 l / \pi r+1 / f(\sigma))
$$

with

$$
f(\sigma)=\frac{1+3.8 \sigma^{5 / 4}}{1-\sigma}
$$

where $r$ is the radius of cylindrical nanochannels and $l$ the length. $N$ is the channel density and $\sigma\left(=\pi r^{2} N\right)$ the membrane porosity. $D_{\mathrm{w}}$ is the diffusion coefficient of probe in water phase. $N, r, \sigma$ and $l$ are measured to be $5.22 \times 10^{16} / \mathrm{m}^{2}, 1.15 \mathrm{~nm}, 21.7 \%$ and $97.5 \mathrm{~nm}$, respectively, by TEM and SEM (Figure S3). The results are summarized in Table S1.

Table S1. Membrane permeability for different probes calculated using eq S19 and

\begin{tabular}{|c|c|c|c|}
\hline \multirow[t]{2}{*}{ Redox Probe } & \multirow{2}{*}{$\begin{array}{c}k_{\mathrm{m}}(\text { eq S19) } \\
\left(\mathrm{mm} \mathrm{s}^{-1}\right)\end{array}$} & \multicolumn{2}{|c|}{$\begin{array}{c}k_{\mathrm{m}} \text { (this work) } \\
\left(\mathrm{mm} \mathrm{s}^{-1}\right)\end{array}$} \\
\hline & & $\mathrm{a}$ & $\mathrm{b}$ \\
\hline $\mathrm{FcMeOH}^{+}$ & 1.67 & 0.18 & 0.33 \\
\hline $\mathrm{Ru}\left(\mathrm{NH}_{3}\right)_{6}{ }^{2+}$ & 2.2 & 1.33 & 2.4 \\
\hline $\mathrm{Ru}(\mathrm{CN})_{6}{ }^{3-}$ & 1.23 & 0.23 & 0 \\
\hline $\mathrm{Ru}(\mathrm{CN})_{6}^{4-}$ & 1.23 & 0.15 & $1.5 \times 10^{-3}$ \\
\hline a $1.0 \mathrm{M} \mathrm{KCl}$ & & & \\
\hline b $0.01 \mathrm{M} \mathrm{KCl}$ & & & \\
\hline
\end{tabular}
model in this work. 


\section{COMSOL model report}

\section{Report date}

Jun 5, 2019 10:05:22 PM

\section{Contents}

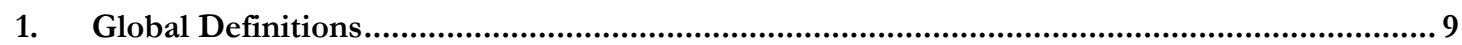

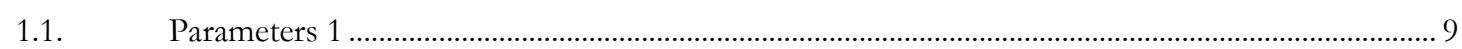

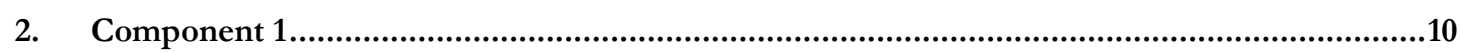

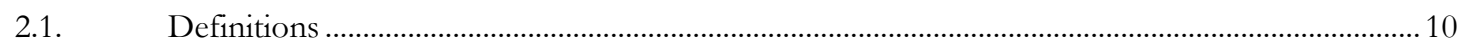

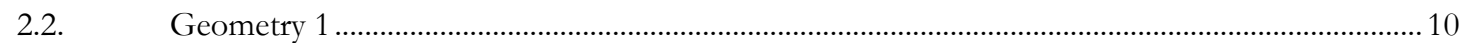

2.3. Transport of Diluted Species............................................................................................................. 11

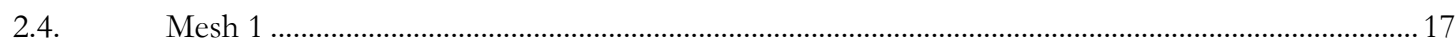

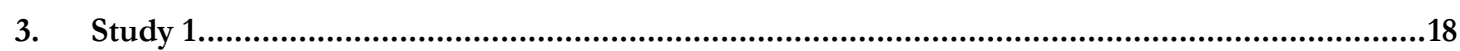

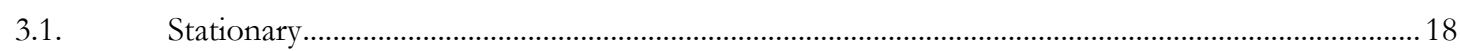

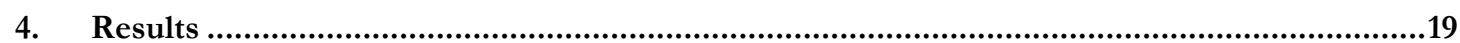

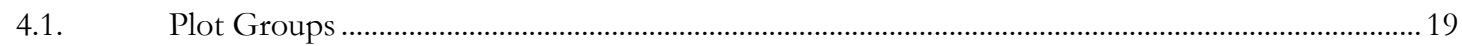




\section{Global Definitions}

Date May 13, 2019 4:44:44 PM

\subsection{Parameters 1}

Table S2. Parameters for the simulation

\begin{tabular}{|c|c|c|c|}
\hline Name & Expression & Value & Description \\
\hline$a$ & $3.5[\mu \mathrm{m}]$ & $3.5 \mathrm{E}-6 \mathrm{~m}$ & "'"'"'"'" \\
\hline RG & 3.5 & 3.5 & "'"'"'"'" \\
\hline $\operatorname{rg}$ & $a^{*} \mathrm{RG}$ & $1.225 \mathrm{E}-5 \mathrm{~m}$ & "'"'"'"'" \\
\hline$d$ & $200[\mu \mathrm{m}]$ & $2 \mathrm{E}-4 \mathrm{~m}$ & "'"'"'"'" \\
\hline $\mathrm{RE}$ & $200 * a$ & $7 \mathrm{E}-4 \mathrm{~m}$ & "'"'"'"'" \\
\hline $\mathrm{CO}$ & $1[\mathrm{mM}]$ & $1 \mathrm{~mol} / \mathrm{m}^{3}$ & "'"'"'"'" \\
\hline $\mathrm{k}$ & $4.5 \mathrm{e}-4[\mathrm{~m} / \mathrm{s}]$ & $4.5 \mathrm{E}-4 \mathrm{~m} / \mathrm{s}$ & "'"'"'"'" \\
\hline $\mathrm{D}$ & $9.4 \mathrm{E}-10\left[\mathrm{~m}^{\wedge} 2 / \mathrm{s}\right]$ & $9.4 \mathrm{E}-10 \mathrm{~m}^{2} / \mathrm{s}$ & "'"'"'"'" \\
\hline $\mathrm{n}$ & 1 & 1 & "'"'"'"'" \\
\hline $\mathrm{F}$ & $96485[\mathrm{C} / \mathrm{mol}]$ & $96485 \mathrm{C} / \mathrm{mol}$ & "'"'"'"'" \\
\hline$b$ & $a * 100$ & $3.5 \mathrm{E}-4 \mathrm{~m}$ & \\
\hline
\end{tabular}




\section{Component 1}

\subsection{Definitions}

\subsubsection{Coordinate Systems}

\section{Boundary System 1}

\begin{tabular}{|l|l|}
\hline Coordinate system type & Boundary system \\
\hline Tag & sys1 \\
\hline
\end{tabular}

\subsection{Geometry 1}

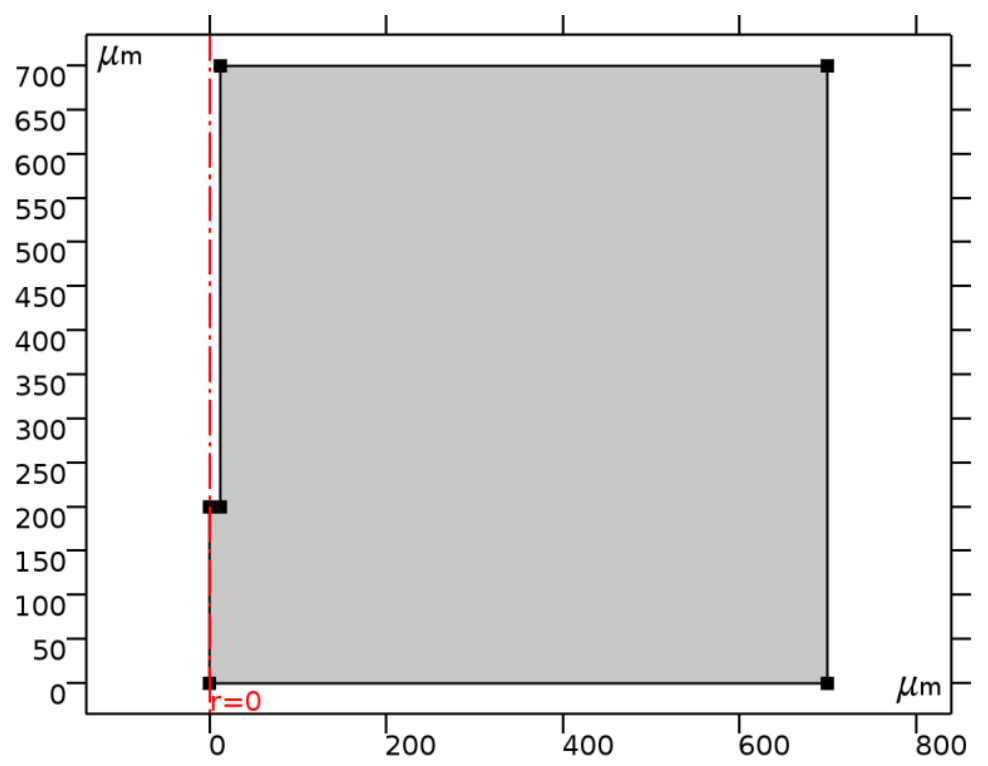

Figure S4. Geometry for the simulation

\section{Units}

\begin{tabular}{|l|l|}
\hline Length unit & $\mu \mathrm{m}$ \\
\hline Angular unit & deg
\end{tabular}




\subsection{Transport of Diluted Species}

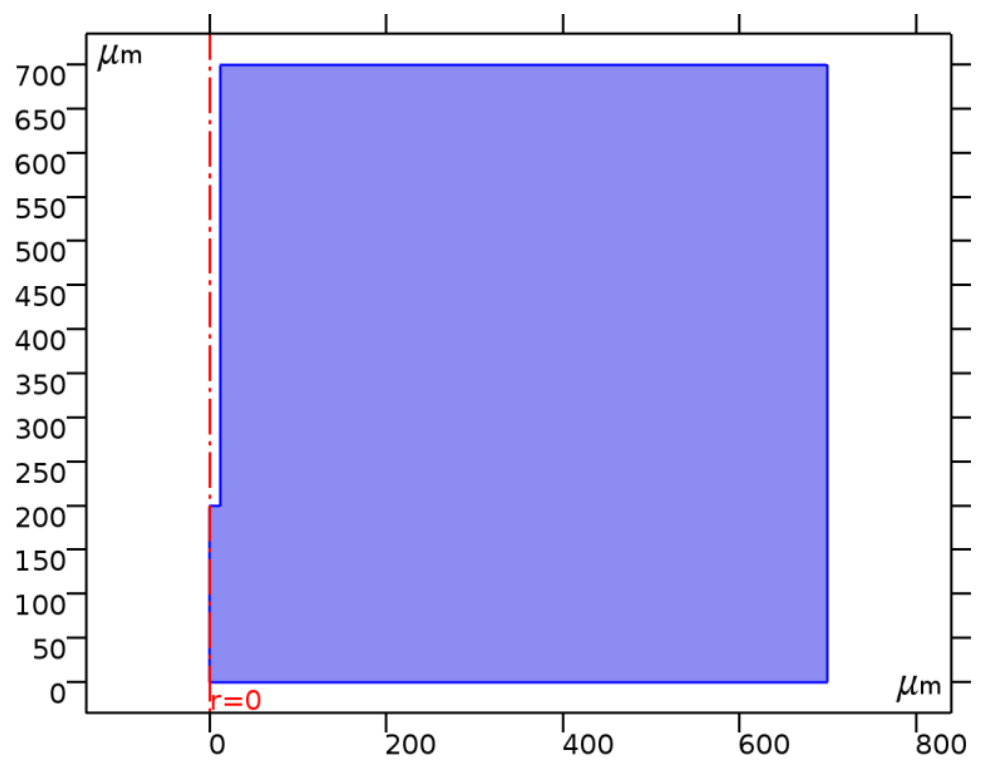

Figure S5. Physics (Transport of Diluted Species) used in the whole simulation domain.

Equations

$\nabla \cdot\left(-D_{i} \nabla c_{i}\right)=R_{i}$

$\mathbf{N}_{i}=-D_{i} \nabla c_{i}$

Table S3. Settings

\begin{tabular}{|l|l|}
\hline Description & Value \\
\hline Concentration & Linear \\
\hline Compute boundary fluxes & On \\
\hline Apply smoothing to boundary fluxes & On \\
\hline Value type when using splitting of complex variables & Real \\
\hline Convection & Off \\
\hline Migration in electric field & Off \\
\hline Mass transfer in porous media & Off \\
\hline Streamline diffusion & On \\
\hline Crosswind diffusion & On \\
\hline Equation residual & Approximate residual \\
\hline Crosswind diffusion type for free flow & Do Carmo and Galeão \\
\hline Convective term & Nonconservative form \\
\hline
\end{tabular}

Table S4. Variables

\begin{tabular}{|c|c|c|c|c|}
\hline Name & Expression & Unit & Description & Selection \\
\hline tds.R_c & 0 & $\mathrm{~mol} /\left(\mathrm{m}^{3} \cdot \mathrm{s}\right)$ & Total & Domain 1 \\
\hline
\end{tabular}




\begin{tabular}{|c|c|c|c|c|}
\hline Name & Expression & Unit & Description & Selection \\
\hline & & & expression & \\
\hline tds.epsilon_p & 1 & 1 & Porosity & Domain 1 \\
\hline tds.theta & tds.epsilon_p & 1 & $\begin{array}{l}\text { Liquid volume } \\
\text { fraction }\end{array}$ & Domain 1 \\
\hline tds.av & 0 & 1 & $\begin{array}{l}\text { Gas volume } \\
\text { fraction }\end{array}$ & Domain 1 \\
\hline tds.nr & $\operatorname{dnr}$ & 1 & $\begin{array}{l}\text { Normal } \\
\text { vector, } \\
\text { component }\end{array}$ & $\begin{array}{l}\text { Boundaries } \\
1-7\end{array}$ \\
\hline tds.nphi & 0 & 1 & $\begin{array}{l}\text { Normal } \\
\text { vector, phi } \\
\text { component }\end{array}$ & $\begin{array}{l}\text { Boundaries } \\
1-7\end{array}$ \\
\hline tds.nz & $\operatorname{dnz}$ & 1 & $\begin{array}{l}\text { Normal } \\
\text { vector, } \quad \mathrm{z} \\
\text { component }\end{array}$ & $\begin{array}{l}\text { Boundaries } \\
1-7\end{array}$ \\
\hline tds.nrmesh & dnrmesh & 1 & $\begin{array}{l}\text { Normal vector } \\
\text { (mesh), r } \\
\text { component }\end{array}$ & $\begin{array}{l}\text { Boundaries } \\
1-7\end{array}$ \\
\hline tds.nphimesh & 0 & 1 & $\begin{array}{l}\text { Normal vector } \\
\text { (mesh), phi } \\
\text { component }\end{array}$ & $\begin{array}{l}\text { Boundaries } \\
1-7\end{array}$ \\
\hline tds.nzmesh & dnzmesh & 1 & $\begin{array}{l}\text { Normal vector } \\
\text { (mesh), z } \\
\text { component }\end{array}$ & $\begin{array}{l}\text { Boundaries } \\
1-7\end{array}$ \\
\hline tds.nrc & $\begin{array}{l}\text { root.nrc/sqrt(root.nrc }{ }^{\wedge} 2+\text { root. } \\
\left.\mathrm{nzc}^{\wedge} 2+\mathrm{eps}\right)\end{array}$ & 1 & $\begin{array}{l}\text { Normal } \\
\text { vector, } \\
\text { component }\end{array}$ & $\begin{array}{l}\text { Boundaries } \\
1-7\end{array}$ \\
\hline tds.nphic & 0 & 1 & $\begin{array}{l}\text { Normal } \\
\text { vector, phi } \\
\text { component }\end{array}$ & $\begin{array}{l}\text { Boundaries } \\
1-7\end{array}$ \\
\hline tds.nzc & $\begin{array}{l}\text { root.nzc/sqrt(root.nrc }{ }^{\wedge} 2+\text { root. } \\
\left.\text { nzc }^{\wedge} 2+\text { eps }\right)\end{array}$ & 1 & $\begin{array}{l}\text { Normal } \\
\text { vector, } \\
\text { component }\end{array}$ & $\begin{array}{l}\text { Boundaries } \\
1-7\end{array}$ \\
\hline
\end{tabular}

\section{Features}

Transport Properties 1

Axial Symmetry 1

No Flux 1 
Initial Values 1

Concentration 1

Concentration 2

Flux 1

\subsubsection{Transport Properties 1}

Equations

$\nabla \cdot\left(-D_{i} \nabla c_{i}\right)=R_{i}$

$\mathbf{N}_{i}=-D_{i} \nabla c_{i}$

Table S5. Settings

\begin{tabular}{|l|l|}
\hline Description & Value \\
\hline Temperature & User defined \\
\hline Temperature & $293.15[\mathrm{~K}]$ \\
\hline Material & None \\
\hline Diffusion coefficient & User defined \\
\hline Diffusion coefficient & $\{\{\mathrm{D}, 0,0\},\{0, \mathrm{D}, 0\},\{0,0, \mathrm{D}\}\}$ \\
\hline
\end{tabular}

Table S6. Variables

\begin{tabular}{|c|c|c|c|c|}
\hline Name & Expression & Unit & Description & Selection \\
\hline domflux.cr & $2 *$ tds.dflux_cr*ai ${ }^{*}{ }_{r}$ & $\mathrm{~mol} /(\mathrm{m} \cdot \mathrm{s})$ & $\begin{array}{l}\text { Domain flux, r } \\
\text { component }\end{array}$ & Domain 1 \\
\hline domflux.cz & $2 *$ tds.dflux_cz*pi*r & $\mathrm{mol} /(\mathrm{m} \cdot \mathrm{s})$ & $\begin{array}{l}\text { Domain flux, z } \\
\text { component }\end{array}$ & Domain 1 \\
\hline tds.ndflux_c & tds.bndFlux_c & $\mathrm{mol} /\left(\mathrm{m}^{2} \cdot \mathrm{s}\right)$ & $\begin{array}{l}\text { Normal diffusive } \\
\text { flux }\end{array}$ & $\begin{array}{l}\text { Boundaries } \\
2-7\end{array}$ \\
\hline tds.ntflux_c & tds.bndFlux_c & $\mathrm{mol} /\left(\mathrm{m}^{2} \cdot \mathrm{s}\right)$ & Normal total flux & $\begin{array}{l}\text { Boundaries } \\
2-7\end{array}$ \\
\hline tds.D_crr & $\mathrm{D}$ & $\mathrm{m}^{2} / \mathrm{s}$ & $\begin{array}{l}\text { Diffusion } \\
\text { coefficient, } \quad \mathrm{rr} \\
\text { component }\end{array}$ & Domain 1 \\
\hline tds.D_cphir & 0 & $\mathrm{~m}^{2} / \mathrm{s}$ & $\begin{array}{l}\text { Diffusion } \\
\text { coefficient, phir } \\
\text { component }\end{array}$ & Domain 1 \\
\hline tds.D_czr & 0 & $\mathrm{~m}^{2} / \mathrm{s}$ & $\begin{array}{l}\text { Diffusion } \\
\text { coefficient, } \quad \mathrm{zr} \\
\text { component }\end{array}$ & Domain 1 \\
\hline tds.D_crphi & 0 & $\mathrm{~m}^{2} / \mathrm{s}$ & Diffusion & Domain 1 \\
\hline
\end{tabular}




\begin{tabular}{|c|c|c|c|c|}
\hline Name & Expression & Unit & Description & Selection \\
\hline & & & $\begin{array}{l}\text { coefficient, rphi } \\
\text { component }\end{array}$ & \\
\hline tds.D_cphiphi & $\mathrm{D}$ & $\mathrm{m}^{2} / \mathrm{s}$ & $\begin{array}{l}\text { Diffusion } \\
\text { coefficient, } \\
\text { phiphi } \\
\text { component }\end{array}$ & Domain 1 \\
\hline tds.D_czphi & 0 & $\mathrm{~m}^{2} / \mathrm{s}$ & $\begin{array}{l}\text { Diffusion } \\
\text { coefficient, zphi } \\
\text { component }\end{array}$ & Domain 1 \\
\hline tds.D_crz & 0 & $\mathrm{~m}^{2} / \mathrm{s}$ & $\begin{array}{l}\text { Diffusion } \\
\text { coefficient, rz } \\
\text { component }\end{array}$ & Domain 1 \\
\hline tds.D_cphiz & 0 & $\mathrm{~m}^{2} / \mathrm{s}$ & $\begin{array}{l}\text { Diffusion } \\
\text { coefficient, phiz } \\
\text { component }\end{array}$ & Domain 1 \\
\hline tds.D_czz & $\mathrm{D}$ & $\mathrm{m}^{2} / \mathrm{s}$ & $\begin{array}{l}\text { Diffusion } \\
\text { coefficient, } \quad \mathrm{zz} \\
\text { component }\end{array}$ & Domain 1 \\
\hline tds.Dav_c & $0.5^{*}\left(\mathrm{tds} . \mathrm{D} \_\mathrm{crr}+\mathrm{tds} . \mathrm{D} \_\mathrm{czz}\right)$ & $\mathrm{m}^{2} / \mathrm{s}$ & $\begin{array}{l}\text { Average } \\
\text { diffusion } \\
\text { coefficient }\end{array}$ & Domain 1 \\
\hline tds.tflux_cr & tds.dflux_cr & $\mathrm{mol} /\left(\mathrm{m}^{2} \cdot \mathrm{s}\right)$ & $\begin{array}{l}\text { Total flux, r } \\
\text { component }\end{array}$ & Domain 1 \\
\hline tds.tflux_cphi & tds.dflux_cphi & $\mathrm{mol} /\left(\mathrm{m}^{2} \cdot \mathrm{s}\right)$ & $\begin{array}{l}\text { Total flux, phi } \\
\text { component }\end{array}$ & Domain 1 \\
\hline tds.tflux_cz & tds.dflux_cz & $\mathrm{mol} /\left(\mathrm{m}^{2} \cdot \mathrm{s}\right)$ & $\begin{array}{l}\text { Total flux, } \mathrm{z} \\
\text { component }\end{array}$ & Domain 1 \\
\hline tds.dfluxMag_c & $\begin{array}{l}\text { sqrt(tds.dflux_cr^2+tds.dflu } \\
\text { x_cphi^2+tds.dflux_cz} 2)\end{array}$ & $\mathrm{mol} /\left(\mathrm{m}^{2} \cdot \mathrm{s}\right)$ & $\begin{array}{l}\text { Diffusive flux } \\
\text { magnitude }\end{array}$ & Domain 1 \\
\hline tds.tfluxMag_c & $\begin{array}{l}\text { sqrt(tds.tflux_cr^2+tds.tflux } \\
\text { _cphi^2+tds.tflux_cz^2) }\end{array}$ & $\mathrm{mol} /\left(\mathrm{m}^{2} \cdot \mathrm{s}\right)$ & $\begin{array}{l}\text { Total flux } \\
\text { magnitude }\end{array}$ & Domain 1 \\
\hline tds.dflux_cr & -tds.D_crr*cr-tds.D_crz*cz & $\mathrm{mol} /\left(\mathrm{m}^{2} \cdot \mathrm{s}\right)$ & $\begin{array}{l}\text { Diffusive flux, } \mathrm{r} \\
\text { component }\end{array}$ & Domain 1 \\
\hline tds.dflux_cphi & $\begin{array}{l}\text {-tds.D_cphir*cr- } \\
\text { tds.D_cphiz*cz }\end{array}$ & $\mathrm{mol} /\left(\mathrm{m}^{2} \cdot \mathrm{s}\right)$ & $\begin{array}{l}\text { Diffusive flux, } \\
\text { phi component }\end{array}$ & Domain 1 \\
\hline tds.dflux_cz & -tds.D_czr*cr-tds.D_czz*cz & $\mathrm{mol} /\left(\mathrm{m}^{2} \cdot \mathrm{s}\right)$ & $\begin{array}{l}\text { Diffusive flux, z } \\
\text { component }\end{array}$ & Domain 1 \\
\hline tds.grad_cr & $\mathrm{cr}$ & $\mathrm{mol} / \mathrm{m}^{4}$ & Concentration & Domain 1 \\
\hline
\end{tabular}




\begin{tabular}{|c|c|c|c|c|}
\hline Name & Expression & Unit & Description & Selection \\
\hline & & & $\begin{array}{l}\text { gradient, } \quad \mathrm{r} \\
\text { component }\end{array}$ & \\
\hline tds.grad_cphi & 0 & $\mathrm{~mol} / \mathrm{m}^{4}$ & $\begin{array}{l}\text { Concentration } \\
\text { gradient, phi } \\
\text { component }\end{array}$ & Domain 1 \\
\hline tds.grad_cz & $\mathrm{cz}$ & $\mathrm{mol} / \mathrm{m}^{4}$ & $\begin{array}{l}\text { Concentration } \\
\text { gradient, } \\
\text { component }\end{array}$ & Domain 1 \\
\hline tds.bndFlux_c & $\begin{array}{l}\text { if }(\mathrm{r}>0.001 / \text { sqrt(sqrt(mean }(\mathrm{e} \\
\text { metric } 2))),- \\
0.5^{*} \text { dflux_spatial(c)/(pi*r),N } \\
\mathrm{aN})\end{array}$ & $\mathrm{mol} /\left(\mathrm{m}^{2} \cdot \mathrm{s}\right)$ & Boundary flux & $\begin{array}{l}\text { Boundaries } \\
1-7\end{array}$ \\
\hline tds.helem & $\mathrm{h}$ & $\mathrm{m}$ & Element size & Domain 1 \\
\hline tds.glim_mass & $0.1\left[\mathrm{~mol} / \mathrm{m}^{\wedge} 3\right] /$ tds.helem & $\mathrm{mol} / \mathrm{m}^{4}$ & $\begin{array}{l}\text { Lower gradient } \\
\text { limit }\end{array}$ & Domain 1 \\
\hline tds.Res_c & -tds.R_c & $\mathrm{mol} /\left(\mathrm{m}^{3} \cdot \mathrm{s}\right)$ & $\begin{array}{l}\text { Equation } \\
\text { residual }\end{array}$ & Domain 1 \\
\hline
\end{tabular}

\subsubsection{No Flux 1}

Equations

$-\mathbf{n} \cdot \mathbf{N}_{i}=0$

\subsubsection{Initial Values 1}

Settings

\begin{tabular}{|l|l|}
\hline Description & Value \\
\hline Concentration & $\mathrm{C} 0$ \\
\hline
\end{tabular}

\subsubsection{Concentration 1}

Equations

$c_{i}=c_{0, j}$

Table S7. Settings

\begin{tabular}{|l|l|}
\hline Description & Value \\
\hline Species c & On \\
\hline Concentration & C0 \\
\hline Apply reaction terms on & All physics (symmetric) \\
\hline Use weak constraints & Off \\
\hline
\end{tabular}




\begin{tabular}{|l|l|}
\hline Description & Value \\
\hline Constraint method & Elemental \\
\hline
\end{tabular}

Table S8. Variables

\begin{tabular}{|l|l|l|l|l|}
\hline Name & Expression & Unit & Description & Selection \\
\hline tds.c0_c & C0 & $\mathrm{mol} / \mathrm{m}^{3}$ & Concentration & Boundaries 6-7 \\
\hline
\end{tabular}

\subsubsection{Concentration 2}

Equations

$c_{i}=c_{0, j}$

Table S9. Settings

\begin{tabular}{|l|l|}
\hline Description & Value \\
\hline Species c & On \\
\hline Concentration & 0 \\
\hline Apply reaction terms on & All physics (symmetric) \\
\hline Use weak constraints & Off \\
\hline Constraint method & Elemental \\
\hline
\end{tabular}

Table S10. Variables

\begin{tabular}{|l|l|l|l|l|}
\hline Name & Expression & Unit & Description & Selection \\
\hline tds.c0_c & 0 & $\mathrm{~mol} / \mathrm{m}^{3}$ & Concentration & Boundary 3 \\
\hline
\end{tabular}

\subsubsection{Flux 1}

Equations

$-\mathbf{n} \cdot \mathbf{N}_{i}=N_{0, i}$

Table S11. Settings

\begin{tabular}{|l|l|}
\hline Description & Value \\
\hline Flux type & General inward flux \\
\hline Species c & On \\
\hline Inward flux & $\mathrm{k}^{*}(\mathrm{C} 0-\mathrm{c})$ \\
\hline
\end{tabular}


2.4 Mesh 1

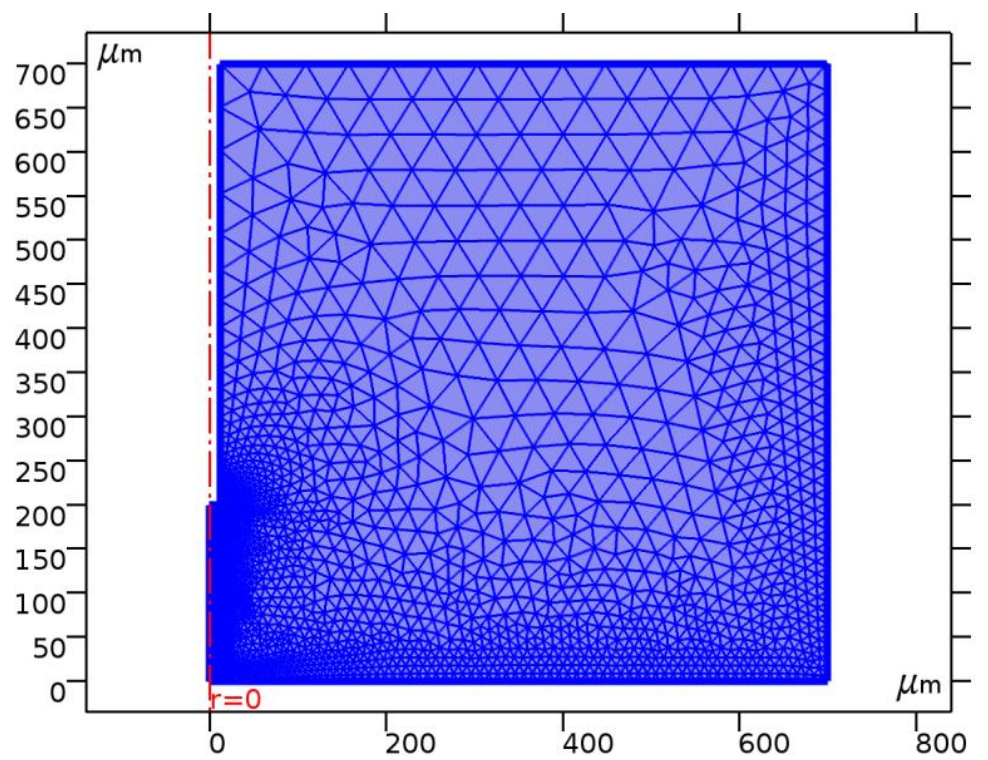

Figure S6. Mesh of the geometry in the simulation 


\section{Study 1}

\subsection{Stationary}

Table S12. Study settings

\begin{tabular}{|l|l|} 
Description & Value \\
\hline Include geometric nonlinearity & Off \\
\hline
\end{tabular}

Table S13. Physics and variables selection

\begin{tabular}{|l|l|}
\hline Physics interface & Discretization \\
\hline Transport of Diluted Species (tds) & physics
\end{tabular}

Table S14. Mesh selection

\begin{tabular}{|l|l|}
\hline Geometry & Mesh \\
\hline Geometry 1 (geom1) & mesh1 \\
\hline
\end{tabular}




\section{Results}

\subsection{Plot Groups}

\subsubsection{Concentration (tds)}

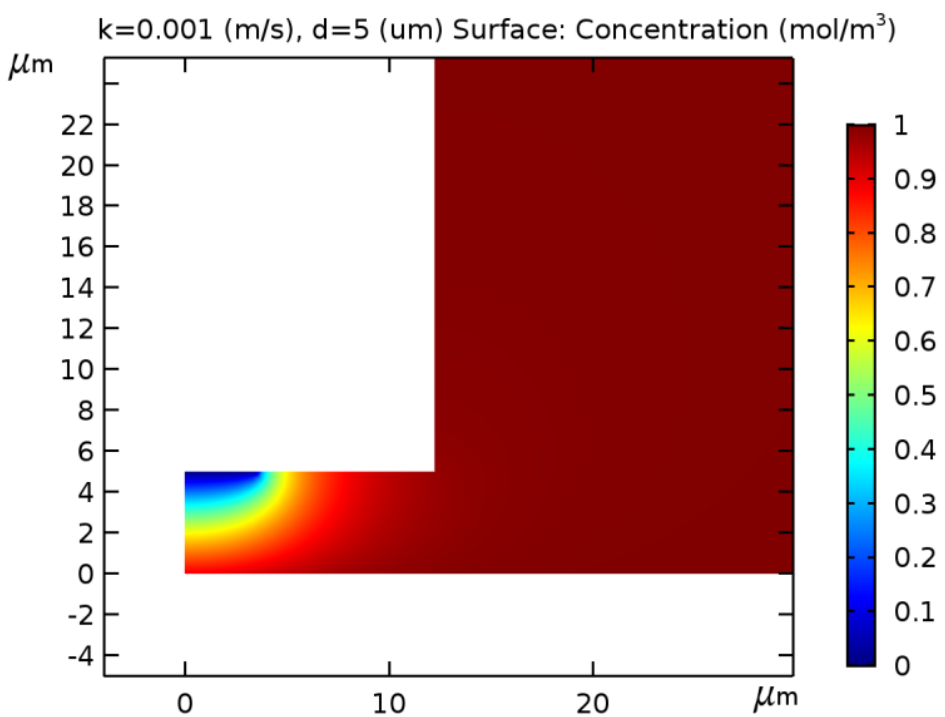

Figure S7. Surface concentration distribution of $R$ between the gap of tip surface and substrate at the distance of $5 \mu \mathrm{m}$.

\subsubsection{Concentration (tds) 2}

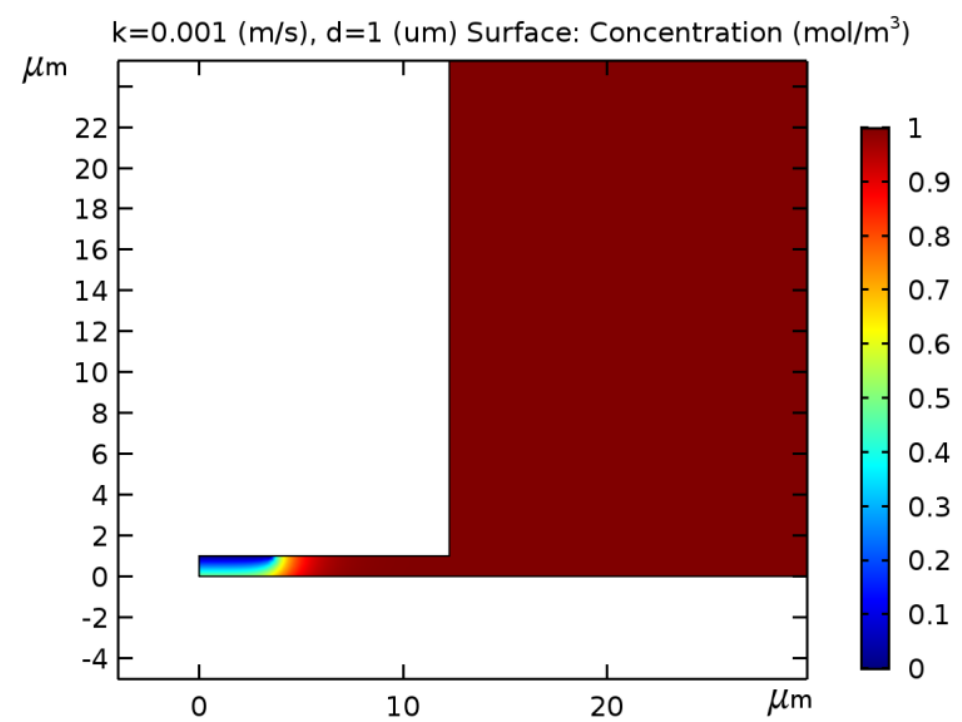

Figure S8. Surface concentration distribution of $\mathrm{R}$ between the gap of tip surface and substrate at the distance of $1 \mu \mathrm{m}$. 


\subsubsection{Concentration (tds) 5}

$k=0.001(\mathrm{~m} / \mathrm{s}), \mathrm{d}=100(\mathrm{um})$ Surface: Concentration $\left(\mathrm{mol} / \mathrm{m}^{3}\right)$

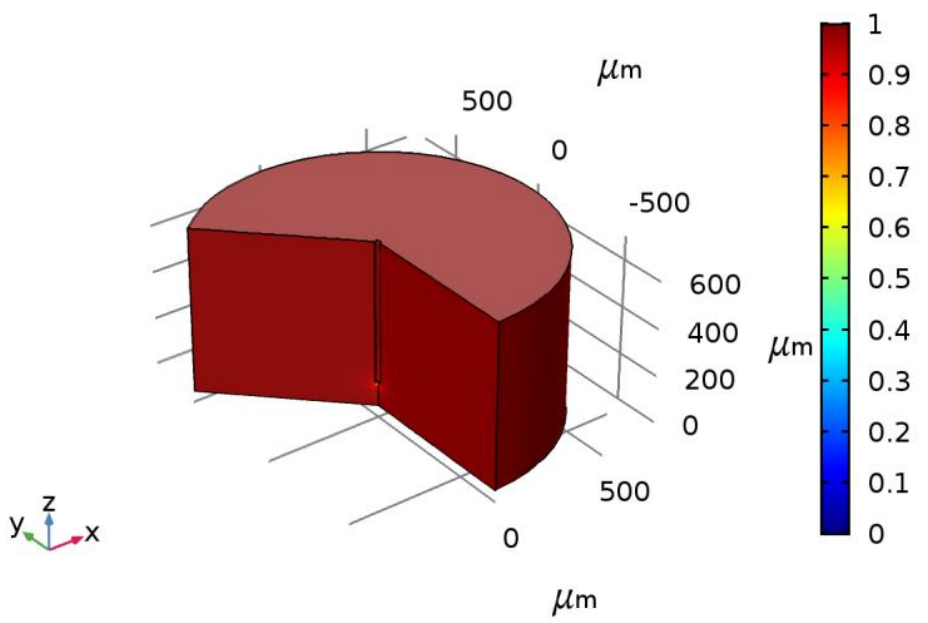

Figure S9. 3D concentration distribution of $\mathrm{R}$ in the simulation domain at the distance of $100 \mu \mathrm{m}$. 